\title{
Cadre des soins de soutien
}

par Margaret I. Fitch

\section{Abrégé}

C'est à 1994 que remonte la formulation initiale du Cadre des soins de soutien pour les soins en oncologie [Supportive Care Framework for Cancer Care] (Fitch, 1994). Ce cadre avait été conçu comme outil destiné aux professionnels des soins en cancer et aux gestionnaires de programmes qui pouvaient s'en servir pour conceptualiser le type d'aide dont leurs patients en cancérologie peuvent avoir besoin et l'approche qui convient à la fourniture des services. Depuis lors, le cadre a été présenté et discuté dans divers domaines et employé dans la planification de programmes et de politiques (Fitch, 2000). Récemment, les demandes de diffusion élargie de la description complète du cadre ont afflué à un rythme croissant.

Cet article a pour but de partager le Cadre des soins de soutien pour les soins en oncologie avec le milieu des soins infirmiers en cancérologie. Ce cadre conceptuel pourra constituer un outil des plus utiles pour la planification de programmes et de services, un fondement pour l'organisation d'approches pédagogiques en soins en cancérologie ou un modèle étayant les projets de recherche.

\section{Introduction}

La formulation initiale du Cadre des soins de soutien pour les soins en oncologie [Supportive Care Framework for Cancer Care] remonte à 1994 (Fitch, 1994). Ce cadre avait été conçu comme outil destiné aux professionnels des soins en cancer et aux gestionnaires de programmes qui pouvaient s'en servir pour conceptualiser le type d'aide dont leurs patients en cancérologie peuvent avoir besoin et l'approche qui convient à la fourniture des services. Depuis lors, le cadre a été présenté et discuté dans divers domaines et employé dans la planification de programmes et de politiques (Fitch, 2000). Récemment, les demandes de diffusion élargie de la description complète du cadre ont afflué à un rythme croissant.

Cet article a pour but de partager le Cadre des soins de soutien pour les soins en oncologie avec le milieu des soins infirmiers en cancérologie. Ce cadre conceptuel pourra constituer un outil des plus utiles pour la planification de programmes et de services, un fondement pour l'organisation d'approches pédagogiques en soins en cancérologie ou un modèle étayant les projets de recherche. Cet article met en relief les fondements théoriques du cadre. Un autre article visant à illustrer les diverses applications du cadre est en cours de préparation.

\section{Contexte}

Les réflexions initiales entourant le Cadre des soins de soutien pour les soins en oncologie sont issues des travaux d'un groupe de travail établi par la Fondation ontarienne pour la recherche en cancérologie et le traitement du cancer (Fitch, 1994). Ce groupe de travail se composait d'experts en soins aux patients atteints de cancer et leur famille représentant un échantillon des disciplines (soins infirmiers, oncoradiologie et oncologie générale, travail social, psychologie, pharmacie, bénévoles et survivants du cancer). Le cadre a été élaboré à partir de l'expérience et de l'expertise collectives de ces intervenants et s'est inspiré des écrits scientifiques généraux sur l'adaptation et le coping.

Margaret Fitch, Centre régional de cancérologie de TorontoSunnybrook, Toronto,ON, courriel : marg.fitch@sunnybrook.ca
Les concepts inclus dans le cadre ont, depuis, été validés au moyen d'entrevues détaillées réalisées auprès de patients et de survivants à propos de leurs expériences face au cancer, à son traitement et de la vie en présence des répercussions du traitement (Gould, James, Gray et Fitch, 2003; Gray, Greenberg, Fitch, Sawka et Hampson, 1998; Gray, Fitch, Davis et Phillips, 1997; Gray, Fitch, Phillips et Labrecque, 2000; Fitch, Deane et Howell, 2003); au moyen de sondages auprès des patients au sujet de leurs besoins et de leur satisfaction à l'égard des soins reçus (Ashbury, Findley, Reynolds et McKerracher, 1998); et au moyen de discussions avec des prestataires de soins en cancérologie relativement à l'expérience qu'ils ont de la satisfaction des besoins des patients (Supportive Care Steering Group, 2004; Fitch et Turner, 2004). Les concepts figurent régulièrement dans la documentation scientifique et, depuis une décennie, constituent le point de mire d'initiatives de recherche de plus en plus nombreuses (Institut national du cancer du Canada, 2005). Dernièrement, les concepts ont été incorporés dans la Stratégie canadienne de lutte contre le cancer (SCLC) et ont orienté les activités d'un groupe d'intervention baptisé Réorientation des efforts (SCLC, 2002). Ce groupe a rédigé une vision pour un système de soins en cancérologie qui décrit un système dans lequel les ressources sont réparties de manière équilibrée afin que les soins prodigués, le soient avec compassion, soient complets et axés sur la personne atteinte. Selon cette vision, ce système a la capacité de répondre aux besoins en soins de soutien des patients atteints de cancer et des survivants.

Cet article présente le Cadre des soins de soutien ainsi que des références scientifiques qui orienteront les lectures plus approfondies sur le sujet. Le cadre devrait être d'une grande utilité à la praticienne individuelle dans son exercice quotidien ainsi qu'à la gestionnaire de programmes se livrant à la mise au point et à l'évaluation de la prestation de services de qualité. Les concepts inhérents au cadre et sa philosophie sous-jacente fournissent un mécanisme qui permet d'envisager et de réaliser l'évaluation infirmière d'un patient individuel et de déterminer les interventions qu'il convient d'examiner dans le cadre du plan de soins. Les concepts peuvent également guider la prise de décisions concernant la gamme de services qui devraient être mis à la disposition des patients atteints de cancer et de leurs proches. Ils peuvent aussi servir à encadrer les programmes d'éducation et de recherche.

\section{Cadre des soins de soutien}

Le Cadre des soins de soutien pour les soins en oncologie se base sur les concepts de besoins humains, l'évaluation cognitive, le coping et l'adaptation pour conceptualiser la manière dont les êtres humains éprouvent le cancer et y font face. L'article mettra en relief tous ces aspects et leur application à l'expérience du cancer.

\section{Besoins humains}

Les êtres humains ne sont pas uniquement des entités physiques. Leur vie a également des facettes émotionnelles, psychologiques, sociales et spirituelles. Les êtres humains agissent à longueur de journée afin de satisfaire leurs besoins dans chacun de ces domaines (Maslow, 1998). Ils se nourrissent, se reposent, dorment, travaillent, jouent et mettent au point des manières de s'adapter au monde et aux changements qui surviennent. À mesure qu'ils progressent vers l'âge adulte, les êtres humains développent toute une palette de compétences leur permettant de prendre soins d'eux-mêmes et de leurs propres besoins.

Lors de la survenue d'événements de la vie et de changements, il peut arriver que la manière habituelle de satisfaire les besoins journaliers ne soit d'aucune efficacité (Dudas et Carlson, 1988). Les besoins de la personne ne peuvent pas être satisfaits de la même façon 
ou en faisant appel aux approches usuelles. Une situation particulière peut engendrer de nouvelles exigences et des anxiétés peuvent commencer à apparaître; des sentiments de vulnérabilité peuvent naître ainsi qu'un sentiment de perte de contrôle sur la situation et qu'une incertitude sur ce qu'il convient de faire. Il se peut que la personne soit obligée, pour répondre à ses besoins, d'accéder à de nouvelles informations, d'apprendre de nouvelles habiletés ou de rechercher l'aide d'autrui. Ces interventions peuvent représenter un véritable défi durant une période de détresse et aggraver le fardeau qui pèse sur la personne (Taylor, 1983). Si les besoins demeurent insatisfaits, la personne continue d'éprouver une détresse émotionnelle et de rencontrer des difficultés.

\section{Évaluation cognitive}

On constate une vaste différence dans la façon dont les individus réagissent à un événement ou une situation de vie particulier(ère). Ainsi, il n'existe pas deux réactions complètement identiques à une même situation. La réponse à un événement varie car celle-ci dépend de l'évaluation cognitive qu'une personne en fait (Lazarus et Folkman, 1984; Park et Folkman, 1997; Folkman et Greer, 2000). L'évaluation cognitive est la démarche selon laquelle la personne réfléchit à un événement, le catégorise ainsi que ses différents aspects et évalue l'effet qu'il exerce sur son bien-être. Il s'agit principalement d'un processus d'évaluation qui se concentre sur la signification et qui survient constamment durant la journée. Lorsque l'individu se trouve confronté à un événement, il se livre à une évaluation primaire. Il juge l'événement non pertinent, bénin, positif ou stressant en répondant à la question « Est-ce pour moi un inconvénient ou un avantage, aujourd'hui ou à l'avenir? » L'évaluation secondaire l'amène à répondre à la question «Que puis-je faire au sujet de cette situation? »Ces évaluations sont faites consciemment ou inconsciemment. Elles sont influencées par des facteurs tels que les connaissances actuelles de la personne, ses expériences passées, son concept de soi, ses besoins, ses attitudes, sa culture et ses grands buts dans la vie. L'individu juge la situation ou lui attribue une signification en utilisant sa perspective personnelle (carte cognitive) et ses idées ou croyances personnelles sur ce qui est important. Des réponses émotionnelles apparaissent ultérieurement et le comportement de l'individu est dicté par l'évaluation qu'il fait de la menace et de l'importance qu'il lui accorde, à la lumière de sa capacité de faire ce qui est exigé par la situation. Si l'individu estime qu'il peut faire peu de choses afin d'éliminer, de minimiser ou de neutraliser la menace pesant sur lui, la détresse émotionnelle monte en flèche.

\section{Coping et adaptation}

Conceptualisé, le coping représente tout ce que fait la personne pour gérer un événement ou une situation et la détresse émotionnelle inhérente (Pearlin et Schooler, 1978). Il s'agit d'un processus dynamique concernant aussi bien les contrariétés quotidiennes que les événements menaçant l'existence. Les êtres humains utilisent un large éventail de stratégies de coping dans leur vie quotidienne alors qu'ils s'efforcent de répondre à leurs besoins et de maintenir un sentiment de bien-être dans un environnement en constante évolution. Ces stratégies de coping peuvent être regroupées en fonction du but qu'elles permettent d'atteindre : 1) axées sur la résolution de problème : gérer ou altérer la situation (le problème) qui provoque la détresse; 2) axées sur les émotions : réguler la réponse émotionnelle à la situation (Lazarus et Folkman, 1984; Parker et Endler, 1996). La sélection des stratégies de coping dans une situation donnée dépend de l'évaluation que l'individu a faite de la situation ou de la signification qu'il y perçoit.

Il est difficile de prédire la combinaison particulière de stratégies de coping qu'un individu donné utilisera dans une situation précise (Pearlin et Schooler, 1978; Folkman et
Moskowitz, 2000). Il est clair que certains individus peuvent sélectionner les mêmes stratégies pour différentes situations et qu'ils peuvent sélectionner des stratégies différentes pour une même situation à des moments différents. Même en présence d'une même situation, des individus distincts manifesteront des réactions différentes et choisiront des stratégies de coping différentes. Pour bien comprendre la réaction comportementale et émotionnelle d'un individu, il est nécessaire de bien saisir l'interprétation ou l'évaluation que cet individu fait de la situation. Les individus réagissent à la signification de la situation à leurs yeux, selon leur propre perspective.

De manière générale, l'ajustement à un événement est lié à quatre ressources personnelles : a) la capacité de voir dans le changement une opportunité et un défi, b) pouvoir changer de stratégies de coping et en utiliser de nouvelles quand les stratégies habituelles ne donnent pas de résultats, c) pouvoir cerner les aspects de l'événement sur lesquels il est possible d'exercer un contrôle personnel et d) accéder aux ressources nécessaires (Bjorck et coll., 1999; Thompson et Collins, 1995; Pollock, Christian et Sands, 1990). L'ajustement aux événements de la vie est une démarche qui se façonne au fil du temps et qui est influencée par des facteurs

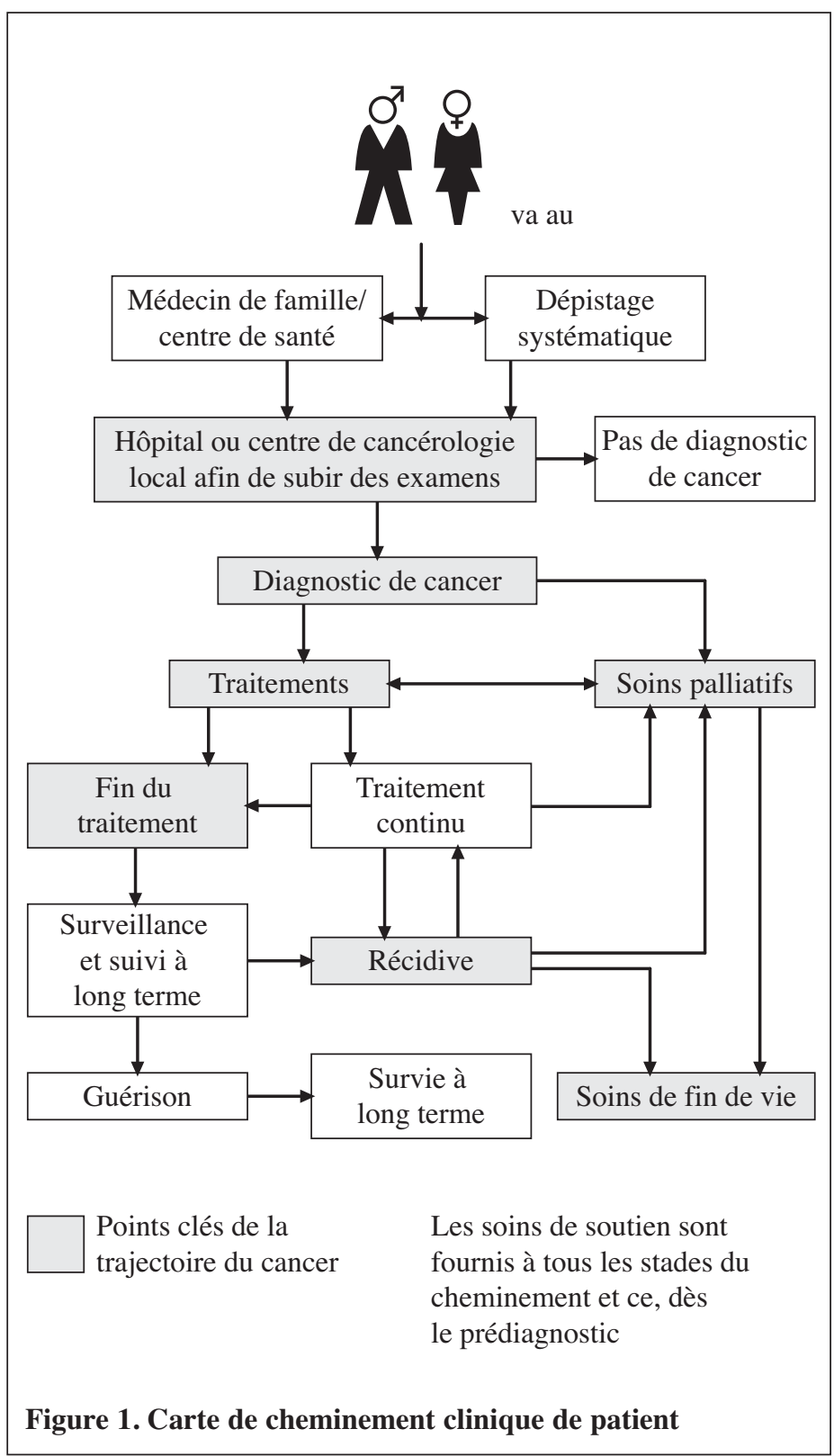


comme le soutien social, les ressources disponibles et l'accès à l'information. Un ajustement efficace suppose une adaptation à l'environnement (physique et social), l'exécution réussie des tâches (demandes) associées à la croissance et au perfectionnement et enfin, la gestion des problèmes ou des demandes accompagnant les situations de vie.

Étant donnée la nature changeante des besoins, la variation des réponses humaines à la vie de tous les jours et aux événements imprévus et la complexité des comportements d'adaptation, il peut s'avérer ardu de trouver le cheminement à suivre pour aider la personne ou d'intervenir afin d'aider la personne à s'ajuster. Les buts recherchés ou les résultats anticipés par la personne peuvent être uniques en leur genre. Pourtant, en ce qui a trait au résultat de toute intervention, il vaut mieux juger le degré de réussite en fonction des buts recherchés et des valeurs de la personne (Lazarus et Folkman, 1984). Il est nécessaire que les interventions conviennent aux buts et valeurs individuels ou que leur personnalisation prenne ces derniers en compte. Quoique les résultats puissent être définis ou désignés d'une manière universelle ou courante (p. ex. qualité de vie, satisfaction, bien-être, fonctionnement optimal), l'interprétation particulière d'un résultat est susceptible de varier d'une personne à l'autre. Ce qui est exigé et utile pour une personne peut ne pas l'être pour une autre. Par exemple, un individu aimera parler avec d'autres personnes afin d'apprendre comment il peut composer avec une situation tandis qu'un autre préférera faire des lectures à cette même fin. À titre d'autre exemple, un individu peut se procurer du soutien en assistant aux réunions d'un groupe de soutien par les pairs alors qu'un autre trouvera ce soutien en plaçant un appel téléphonique interurbain. Il est donc primordial d'explorer avec l'individu les options en matière d'intervention, son désir d'obtenir une assistance et la meilleure façon de lui fournir l'aide requise. Il se peut qu'il faille répéter, au fil du temps, cette exploration ou conversation axée sur la personne.

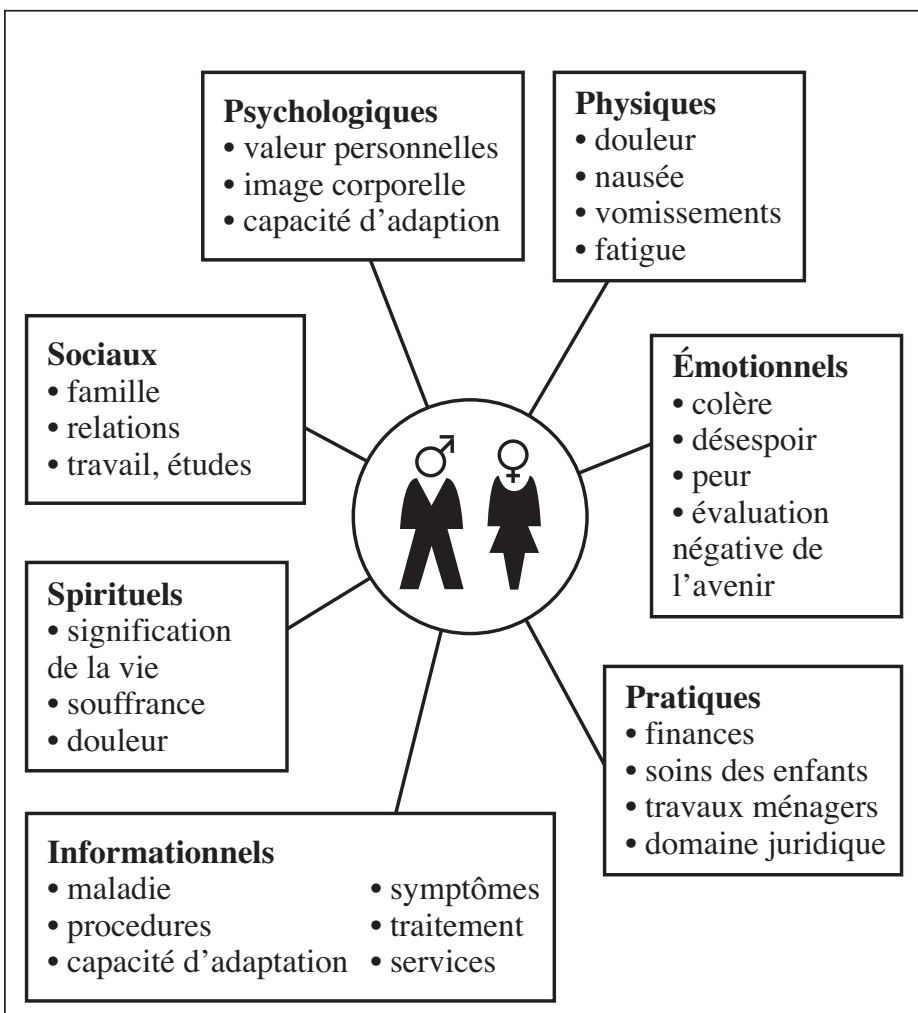

Figure 2. Exemples de besoins des personnes ayant le cancer

\section{Le cancer en tant qu'événement de vie imprévu}

Le diagnostic de cancer et son traitement peuvent être conceptualisés comme un événement de vie imprévu-ou une série d'événements de vie imprévus - au sein d'une gamme d'expériences liées à la maladie. Chez certains patients, le cancer pourra prendre la forme d'un événement particulier ayant un début et une fin bien déterminés. Chez d'autres, l'expérience du cancer pourra revêtir une nature chronique. Chez tous les patients, il existe une gamme d'événements et d'interactions avec le système des soins en cancérologie qui va du prédiagnostic au suivi en passant par le diagnostic et le traitement. Selon la situation, le suivi peut comprendre la survie à long terme, la récidive de cancer ou le décès des suites de la maladie.

Les personnes peuvent accéder au système de soins en cancérologie à divers points et traverser toute une gamme d'expériences et ce, à des rythmes différents et en suivant des cheminements cliniques distincts (voir la figure 1-Carte de cheminement clinique de patient). Certains y accèdent au stade du dépistage et peuvent ne jamais devoir y accéder de nouveau. D'autres y accèdent au stade du diagnostic et traversent les phases du traitement, de la réadaptation et du suivi. Au stade du suivi, certains patients poursuivront leur vie sans aucune nouvelle manifestation clinique du cancer tandis que d'autres seront touchés par une récidive de la maladie. De plus, certains individus accèdent au système au stade du diagnostic et exigent immédiatement des soins palliatifs. Enfin, les décès provoqués par le cancer demeurent le résultat final pour environ la moitié des personnes diagnostiquées (INCC, 2007). Pour certains, le décès survient relativement peu de temps après le diagnostic; pour d'autres, par contre, il peut se produire après bien des années de vie avec la maladie.

Depuis le moment où un individu pense qu'il y a quelque chose qui ne va pas dans son organisme jusqu'à la fin de l'investigation diagnostique, du traitement et des soins de suivi, la vie avec le cancer est une expérience continuelle pour cet individu et sa famille (Howell, Fitch et Deane, 2003). Les événements associés au cancer font partie intégrante de leur vie quotidienne. Les exigences liées à la maladie en viennent à faire partie de la vie et de la prise de décision de tous les jours. Il arrive souvent que le cancer devienne un spectre omniprésent, et non pas une entité à part que l'on peut facilement mettre de côté et ignorer. Les événements liés au cancer sont interconnectés à la fois pour la personne atteinte et pour sa famille. Ce qui s'est produit antérieurement exerce une influence sur ce qui se produira plus tard; ils vivent une épreuve permanente.

\section{L'impact du cancer dépasse la dimension physique}

Le cancer et son traitement exercent un impact que la personne éprouvera de diverses façons. Outre les changements physiques, le cancer peut provoquer des changements d'ordre émotionnel, social, psychologique, économique et spirituel (Société canadienne du cancer, 1992; Ashbury et coll., 1998; Société canadienne du cancer, 2003). Le fait de composer avec ces changements fait naître une multitude de questions et de défis chez la personne atteinte d'un cancer et les membres de sa famille. Diverses réactions se manifestent : choc, incrédulité, confusion, tristesse, colère, soulagement et résignation (Canadian Association of Psychosocial Oncology, 2003). Beaucoup affirment que la vie n'est plus jamais pareille après qu'ils aient entendu proférer le mot «cancer » à leur sujet. Certains individus font face avec succès à leur diagnostic en faisant appel à des méthodes de coping familières, tandis que d'autres trouvent que les approches habituelles ne leur sont d'aucune utilité. Certains gèrent plutôt bien les défis auxquels ils se trouvent confrontés tandis que d'autres sont aux prises avec des difficultés et une détresse permanentes. Il n'y a pas deux personnes qui manifestent 
exactement les mêmes réactions aux circonstances entourant leur expérience du cancer, et ce, même au sein d'une même famille; en revanche, tous les membres seront touchés à un degré ou à un autre (Kristjanson et Ashcroft, 1994; Yates, 1999).

\section{Les personnes diagnostiquées du cancer et}

leur famille ont un large éventail de besoins

Lorsque le cancer frappe, la capacité de la personne à répondre à ses propres besoins peut être compromise. Un inconfort physique ou un sentiment de crise personnelle peut porter atteinte aux approches que la personne utilise habituellement pour satisfaire ses propres besoins. En outre, lorsqu'une personne est confrontée à une maladie à issue potentiellement fatale, des questions particulières font leur apparition à mesure qu'elle explore diverses idées sur la signification de la vie et sur sa propre mortalité (voir la figure 2).
Tout au long de leur traversée du cancer, les sujets atteints et leurs proches éprouvent des changements de nature physique, émotionnelle, sociale, psychologique, spirituelle, informationnelle et pratique qui ont une incidence sur les besoins éprouvés. De nombreuses études ont décrit l'évolution des besoins des patients atteints de cancer (voir le tableau 1 : Définir les besoins). Les besoins particuliers varieront d'une personne à une autre et aussi chez une même personne, au fil du temps, selon le cheminement de la maladie et le cours du traitement. D'ailleurs, la nature de chaque besoin peut aussi évoluer au fil du temps, et l'intensité de l'impact varie fréquemment durant la maladie (Fitch, 2000). Par exemple, durant la phase précoce de la maladie, le besoin d'information médicale est élevé; le besoin de soutien émotionnel et spirituel peut demeurer relativement constant tout au long de la maladie tandis que le besoin de prise en charge de la douleur et des symptômes peut occuper une place prédominante à mesure que la maladie

Tableau 1. Définir les besoins des personnes atteintes de cancer : catégories de besoins et exemples à l'appui

\begin{tabular}{|c|c|c|c|c|}
\hline Définition des catégories & \multicolumn{4}{|c|}{ Exemples précis de symptômes et besoins liés aux soins de soutien } \\
\hline $\begin{array}{l}\text { Dom. physique } \\
\text { Besoins de jouir d'un confort physique } \\
\text { et d'une absence de douleur, d'une } \\
\text { alimentation optimale et d'être capable } \\
\text { d'exécuter ses fonctions journalières } \\
\text { habituelles (p. ex. activités de la } \\
\text { vie quotidienne) }\end{array}$ & $\begin{array}{l}\text { - douleur } \\
\text { - faiblesse } \\
\text { - fatigue } \\
\text { - anorexie } \\
\text { - nausée } \\
\text { - vomissements } \\
\text { - mobilité } \\
\text { - lymphœedème }\end{array}$ & $\begin{array}{l}\text { - incontinence } \\
\text { - soif } \\
\text { - toux } \\
\text { - dyspnée } \\
\text { - cracher du sang } \\
\text { - perte d'appétit } \\
\text { - perte de cheveux }\end{array}$ & $\begin{array}{l}\text { - impuissan } \\
\text { - troubles d } \\
\text { - difficultés } \\
\text { - difficultés } \\
\text { - changeme } \\
\text { - ménopaus } \\
\text { - difficulté } \\
\text { - fertilité }\end{array}$ & $\begin{array}{l}\text { transit } \\
\text { face à l'apport alimentaire et/ou liquidien } \\
\text { de sommeil } \\
\text { ts de poids } \\
\text { avaler/parler }\end{array}$ \\
\hline $\begin{array}{l}\text { Dom. de l'information } \\
\text { Besoins d'information en vue de } \\
\text { réduire la confusion, l'anxiété et la peur, } \\
\text { d'éclairer la prise de décision de la } \\
\text { personne ou de la famille, et enfin, de } \\
\text { favoriser l'acquisition d'habiletés }\end{array}$ & \multicolumn{3}{|c|}{$\begin{array}{l}\text { - traitement du cancer et ses effets } \\
\text { secondaires, procédures / résultats d'examens } \\
\text { - comment faire face ou gérer les effets secondaires } \\
\text { - processus de soins }\end{array}$} & $\begin{array}{l}\text { - communication avec les } \\
\text { prestataires de soins } \\
\text { - orientation à l'établissement de soins } \\
\text { - ressources éducatives (appropriées) } \\
\text { - aide à la prise de décision }\end{array}$ \\
\hline $\begin{array}{l}\text { Dom. émotionnel } \\
\text { Besoins d'être à l'aise, d'éprouver } \\
\text { un sentiment d'appartenance, de la } \\
\text { compréhension et d'être rassuré durant les } \\
\text { périodes de stress et de bouleversement }\end{array}$ & $\begin{array}{l}\text { - } \text { peur } \\
\text { - détresse } \\
\text { - anxiété } \\
\text { - dépression } \\
\text { - colère }\end{array}$ & \multicolumn{3}{|c|}{$\begin{array}{ll}\text { - culpabilité } & \text { - parler avec un pair } \\
\text { - chagrin } & \text { - sentiment d'impuissance } \\
\text { - abandon } & \text { ' honte / culpabilisation } \\
\text { - évaluation négative de l'avenir } & \bullet \text { isolement }\end{array}$} \\
\hline $\begin{array}{l}\text { Dom. psychologique } \\
\text { Besoins reliés à la capacité de } \\
\text { composer avec l'expérience de la } \\
\text { maladie et ses conséquences, } \\
\text { notamment le besoin d'éprouver } \\
\text { un contrôle de soi optimal et } \\
\text { une estime de soi positive }\end{array}$ & \multicolumn{2}{|c|}{$\begin{array}{l}\text { - changements de style de vie } \\
\text { - problèmes d'ordre sexuel } \\
\text { - diminution de la capacité cognitive } \\
\text { - perte }\end{array}$} & \multicolumn{2}{|c|}{$\begin{array}{l}\text { - perte de contrôle de soi } \\
\text { - dépression majeure } \\
\text { - troubles anxieux } \\
\text { - problèmes liés à l'image de soi } \\
\text { - changements de l'image corporelle } \\
\text { - peur de la récidive }\end{array}$} \\
\hline $\begin{array}{l}\text { Dom. social } \\
\text { Besoins liés aux relations familiales, } \\
\text { à l'acceptation sociale et à l'implication } \\
\text { active dans des relations }\end{array}$ & \multicolumn{4}{|c|}{$\begin{array}{l}\text { - changements de rôles } \\
\text { - difficulté à composer avec les réactions des enfants et des membres de la famille } \\
\text { - relations sociales } \\
\text { - communication interpersonnelle } \\
\text { - dire à autrui qu'on est atteint } \\
\end{array}$} \\
\hline $\begin{array}{l}\text { Dom. spirituel } \\
\text { Besoins liés à la signification de la vie } \\
\text { et à la raison d'être }\end{array}$ & \multicolumn{2}{|c|}{$\begin{array}{l}\text { - pratique des croyances religieuses } \\
\text { - quête de signification } \\
\text { - désespoir existentiel }\end{array}$} & \multicolumn{2}{|c|}{$\begin{array}{l}\text { - examen des valeurs et priorités personnelles } \\
\text { - crise spirituelle et sa résolution } \\
\text { - sentiments de non-espoir }\end{array}$} \\
\hline $\begin{array}{l}\text { Dom. pratique } \\
\text { Besoins d'aide directe en vue d'accomplir } \\
\text { une tâche ou une activité particulière et } \\
\text { réduire ainsi les exigences placées sur la } \\
\text { personne (p. ex. services d'aide familiale, } \\
\text { assistance financière) }\end{array}$ & \multicolumn{2}{|c|}{$\begin{array}{l}\text { - aide ménagère journalière } \\
\text { - magasinage } \\
\text { - transport } \\
\text { - soins des enfants } \\
\text { - prothèses } \\
\text { - déplacements entre le domicile } \\
\text { et les lieux de traitement }\end{array}$} & \multicolumn{2}{|c|}{$\begin{array}{l}\text { - aide pour les activités quotidiennes } \\
\text { - fourniture d'aide à la famille } \\
\text { - stress touchant la famille, les enfants, les parents, etc. } \\
\text { - questions financières } \\
\text { - questions juridiques } \\
\text { - questions relatives à l'emploi, aux études } \\
\text { - planification des menus / préparation des aliments }\end{array}$} \\
\hline
\end{tabular}


évolue. Bien que le stade du cancer et le type de traitement influent sur les symptômes physiques avec lesquels le patient doit composer, les symptômes ne sont pas nécessairement associés à l'intensité de la détresse émotionnelle éprouvée par les individus (Zabora, Blanchard et Smith, 1997). Pour certains, la détresse émotionnelle peut perdurer bien après la disparition des signes cliniques du cancer.

La capacité de la personne à satisfaire ses propres besoins au long de l'expérience du cancer est influencée par divers facteurs. Un inconfort ou une invalidité physique, la détresse émotionnelle ou un sentiment de crise personnelle peut nuire à la capacité d'agir de la personne (Zabora et coll., 1997; Folkman et Green, 2000; Bury, 1982). Les manières habituelles de satisfaire les besoins journaliers peuvent s'en trouver compromis et la personne doit acquérir de nouvelles connaissances, de nouvelles compétences, ou alors trouver des soutiens différents en vue de composer avec les demandes de la situation liée au cancer. Toutefois, rechercher de l'information, acquérir de nouvelles compétences ou tendre la main vers d'autres personnes peuvent s'avérer difficiles lorsqu'apparaissent les stress de l'expérience du cancer. La non-satisfaction des besoins augmente le fardeau de souffrance et de détresse émotionnelle vécue par les patients atteints de cancer (Weisman, 1979; Rosenbaum, 1982; Charmaz, 1991; McLean, 1993; Phillips, 1998; David, 1999; Gray, 2003).

\section{La détresse émotionnelle est une réaction anticipée à la maladie}

La détresse émotionnelle ou la détresse psychosociale constituent une réaction naturelle ou anticipée à une maladie à issue potentiellement mortelle. Elle est éprouvée, à un degré ou à un autre, par tous les patients atteints de cancer. Même ceux qui sont capables de mobiliser leurs propres ressources et de composer efficacement avec le diagnostic en éprouveront à tout le moins une agitation émotionnelle. Quant aux individus qui ne trouvent aucun moyen de répondre à leurs besoins, ils ressentent une détresse émotionnelle continuelle qui ne cesse d'augmenter. La détresse émotionnelle peut atteindre des niveaux si élevés qu'elle peut compromettre la fidélité au traitement, accroître l'utilisation d'autres services de santé et faire grimper les coûts des soins (Holland, 1999; Browne, Arpin, Corey, Fitch et Gaffing, 1990; Zabora, BrintzenhofeSzoc, Curbow, Hooker et Piantadosi, 2001).

Au sein d'un groupe mixte de patients atteints de cancer et traités en clinique externe, jusqu'à $35 \%$ d'entre eux manifestent une détresse émotionnelle importante ou un niveau de détresse tel qu'ils pourraient tirer profit de consultations professionnelles (Zabora et coll., 2001). Lorsque les patients sont regroupés en fonction du stade de leur maladie, la prévalence d'une détresse psychosociale importante varie. Dans une étude, $39 \%$ des patients recevant un traitement actif éprouvaient une détresse psychosociale importante tandis que $79 \%$ des patients recevant des soins palliatifs et $17 \%$ des patients qui n'avaient pas encore de signes cliniques de la maladie en éprouvaient une à des niveaux semblables (Fitch, Vachon, Greenburg et Franssen, 1996). À elle seule, la présence de détresse psychosociale ne révèle pas la raison pour laquelle elle existe et ne permet pas nécessairement d'isoler un type particulier d'intervention. Avant d'établir la ou les intervention(s) appropriée(s), il convient de dialoguer avec la personne pour mettre au jour la raison de sa détresse et le désir qu'elle a de recevoir de l'aide.

\section{L'aide aux personnes atteintes de cancer exige des interventions personnalisées}

Pour trouver la meilleure façon d'aider les patients atteints de cancer à répondre à leurs besoins, il est nécessaire de personnaliser les interventions. L'intervention ou le jeu d'interventions qui est utile pour une personne donnée ne sera pas nécessairement d'une grande utilité pour une autre. Les interventions retenues pour chaque patient en cancérologie doivent correspondre aux besoins personnels de l'individu, à ses buts recherchés et à ses mécanismes de coping. L'ajustement à une maladie à issue potentiellement mortelle et l'adaptation à ses répercussions sont influencés par la perception qu'a l'individu de la situation et par une foule de facteurs tels que le statut socio-économique, les antécédents scolaires, le soutien social, la culture, la religion et le lieu géographique. Il y a aussi le fait que les gens apprennent de diverses manières et qu'ils varient dans la capacité de vivre avec l'incertitude et la détresse émotionnelle. Certains voudront recevoir de l'aide, d'autres non. Et certains pourront décliner l'offre d'aide à un moment donné et l'accepter à un autre.

Les interventions offertes aux individus doivent se fonder sur une évaluation minutieuse des besoins de l'individu dans chaque domaine et être personnalisées. La personnalisation d'une intervention et l'évaluation ultérieure de son succès doivent se baser sur le cadre de référence et sur les buts recherchés du patient individuel (p. ex. l'intervention a-t-elle été suffisamment personnalisée pour convenir à la situation individuelle et aux facteurs personnels, à l'événement particulier, pour ce qui est du patient concerné?). Par exemple, les femmes attribuent une importance différente à l'information que les hommes ne le font et les jeunes femmes souhaitent recevoir des types de soutien qui diffèrent de ceux voulus par les femmes plus âgées (Fitch, 2007). Il faut donc déterminer les résultats des interventions en y incorporant les perspectives du patient. Il ne faut pas qu'ils soient uniquement axés sur les perspectives des professionnels de la santé.

La communication et la fourniture d'information constituent des aspects primordiaux de l'évaluation de situations et de la détermination des interventions appropriées. La communication avec le patient sera gouvernée par des facteurs tels que les normes et pratiques culturelles, les attitudes à l'égard du système de santé et ses prestataires et les expériences précédentes en matière de soins de santé et de cancer (Muzzin, Anderson, Figueredo et Gudelis, 1994). La manière dont la communication se produit entre prestataires de soins et patients peut être perçue comme apportant un soutien et une aide ou, au contraire, comme un fardeau additionnel (Bakker, Fitch, Gray, Reed et Bennett, 2001). Durant des périodes de détresse, il est possible que les individus ne puissent entendre ou bien se souvenir de l'information fournie. En outre, certains individus voudront obtenir de l'information détaillée tandis que d'autres n'en voudront pas; tous peuvent se sentir dépassés par la quantité d'information qu'ils doivent absorber et le langage scientifique utilisé. Essentiellement, les patients atteints de cancer veulent avoir certaines informations à propos de la maladie, des options de traitement, du pronostic probable et des ressources disponibles (Luker, Beaver, Lenster et Owens, 1996). L'information est un élément fondamental d'une prise de décision éclairée mais les individus n'auront pas tous le même désir de s'impliquer activement dans la prise de décision concernant leurs soins (Hack, Degner et Dyck, 1994; Davison, Degner et Morgan, 1995; Davison et Degner, 1997).

Le tableau 2 présente un résumé des résultats sur la proportion de patients atteints de cancer qui pourraient avoir besoin d'aide dans chacun des domaines de besoins. Le siège, le stade du cancer et la modalité de traitement influence le type d'enjeux auxquels les patients doivent faire face. Par exemple, le cancer colorectal traité au moyen de la chirurgie pourrait exiger du patient qu'il s'adapte à une colostomie et la leucémie pourrait l'amener à composer avec une greffe de moelle osseuse. Il convient de tenir un dialogue avec le patient afin de cerner les questions particulières qui le préoccupent et celles pour lesquelles il aimerait recevoir de l'aide. Il importe de reconnaître que tous les patients ne voudront pas nécessairement d'aide à propos d'une question particulière même si elle les préoccupe (Fitch et Steele, 2003). Sans une conversation dirigée axée sur la personne au sujet des types d'aide que la personne souhaite recevoir, les soins fournis pourraient ne pas convenir. Le patient et la 
prestataire de soins doivent établir un partenariat et avoir une idée claire des buts ou des résultats qu'ils cherchent à atteindre. En pratique, cela signifie que le processus d'évaluation se doit de dégager les attentes et les buts de l'individu, dans tous les domaines de besoins, dans le contexte d'une situation existante avant de personnaliser le plan d'intervention en fonction de ces paramètres et en collaboration avec le patient. Ce processus d'évaluation, d'établissement mutuel de buts et de personnalisation des interventions doit nécessairement se produire tout au long de la trajectoire du patient atteint de cancer en vue de réduire la détresse émotionnelle et de renforcer la capacité d'adaptation de la personne (Fitch, 2003; Zabora, Loscalzo et Weber, 2003).

Définition des soins de soutien

Les soins de soutien sont définis comme étant la prestation des services nécessaires aux personnes vivant avec le cancer ou touchées par la maladie afin de satisfaire leurs besoins d'ordre physique, émotionnel, social, psychologique, informationnel, spirituel et pratique durant les phases du diagnostic, du traitement et du suivi, ce qui peut comprendre les questions liés à la survie, aux soins palliatifs et au deuil (Fitch, 1994).

Les soins de soutien englobent l'éventail complet des questions/défis auxquels l'individu fait face alors qu'il éprouve l'impact du diagnostic de cancer et de son traitement et s'efforce de s'adapter à leurs répercussions. En d'autres mots, il s'agit d'un concept global qui décrit l'ensemble de l'aide dont pourraient avoir besoin les patients atteints de cancer et leurs proches au-delà de leurs interventions de médecine, de chirurgie ou de radiothérapie. Les interventions de soutien, quelle que soit leur diversité ou les organismes qui les fournissent, ont pour but d'aider l'individu à s'adapter avec ce qui lui arrive et de maintenir sa qualité de vie. Les soins de soutien visent à améliorer et préserver la qualité de vie, l'autonomie et la dignité des personnes atteintes de cancer et celles touchées par la maladie. Leur but est l'autonomisation et l'optimisation du bien-être intégral.

Pour être efficaces, les soins de soutien doivent être basés sur les besoins de l'individu dans le contexte de sa situation unique en son genre et convenir à ses besoins. Les interventions en soins de soutien doivent être personnalisées aux buts recherchés de l'individu. Ce sont les besoins du patient qui déterminent la nature des soins de soutien qui seront fournis et non pas les bons vœux de la prestataire de soins. L'idée centrale derrière le choix des interventions est de collaborer avec le patient en vue de dégager les approches particulières requises afin de satisfaire ses besoins uniques en leur genre.

\section{Tableau 2. Proportion de patients atteints de cancer nécessitant divers types d'aide ${ }^{1,2}$}

\begin{tabular}{l|l}
\hline Type de besoin & Proportion des patients atteints de cancer nécessitant de l'aide \\
\hline $\begin{array}{l}\text { Détresse liée aux } \\
\text { symptômes/à la douleur } \\
\text { (physique) }\end{array}$ & $\begin{array}{l}\text { La proportion des patients éprouvant des difficultés au niveau des symptômes / de la douleur variera en } \\
\text { fonction du siège et du stade de la maladie. Dans trois études canadiennes des besoins des patients externes } \\
\text { en cancérologie, les proportions suivantes ont été constatées pour les symptômes le plus fréquemment } \\
\text { mentionnés pour le mois précédent : fatigue } 28 \%-40 \% \text {; douleur 21\%-32\%; troubles du sommeil } 16 \%-33 \% \\
\text { nausée } 19 \%-31 \% .\end{array}$ \\
\hline
\end{tabular}

Émotionnel

Tous les patients ont besoin, à un degré ou à un autre, de soutien émotionnel. Le défi est d'établir une bonne correspondance entre l'individu et le type de soutien qui lui convient le mieux (p. ex. pair, groupe, professionnel de la santé).

Cognitif

Psychosocial

20\%-30\% des patients atteints de cancer éprouvent une détresse psychosociale importante et

Spirituel bénéficieraient donc de l'intervention de professionnels de la santé. La source de la détresse n'est pas manifeste tant qu'une évaluation n'a pas été réalisée. Les enjeux existants peuvent se rapporter à des changements touchant l'image corporelle, l'estime de soi, la foi, la signification de la vie, le désespoir, la culpabilité, etc.

Alimentaire

$31 \%-87 \%$ des patients subissent une perte de poids durant le traitement. La perte de poids est un indicateur pronostique majeur de la survie et de la réponse au traitement. 47\%-61\% des patients nécessitaient une intervention de la part d'une diététiste.

Orthophonie Chez tous les patients subissant une chirurgie compromettant la déglutition, le langage pourrait être affecté.

Assistance pratique :

Transport

Soins de jour

questions juridiques, financières

Information

Ces questions dépendent grandement de l'âge de l'individu et de ses proches et de la situation familiale et financière. Elles pourraient aussi comprendre des enjeux liés au travail, aux responsabilités ménagères, aux études, aux soins des enfants, aux soins de relève, etc.

propice au dialogue et de fournir aux patients des occasions de poser des questions. Ils ont besoin d'information sur le cancer, les processus de soins, les symptômes, les procédures, le traitement, les habiletés d'adaptation, les services disponibles, etc.

Maladie mentale

$10 \%-20 \%$ de tous les patients en oncologie sont atteints d'une maladie mentale grave telle que dépression majeure, trouble anxieux, délire ou démence. La réussite du traitement de la dépression majeure requerra probablement des médicaments.

${ }^{1}$ ne tient pas compte des différents sièges/stages de la maladie

${ }^{2}$ Références : Steele, Fitch, Alderson, Barrow, Hurst et Doyle (2004); Sanson-Fisher et coll. (2000); Singer, Margin et Kelner (1999);

Fitch, Gray, DePetrillo, Franssen et Howell (1999); Whelan et coll. (1997); Degner et Sloan (1995) 
La fourniture des soins de soutien

Selon la philosophie des soins de soutien, il est nécessaire de faire appel à toute une gamme d'expertise pour fournir des soins complets aux patients atteints de cancer et aux membres de leur famille. Les soins de soutien ne sont pas un privilège exclusif d'une discipline ou d'un organisme. Divers aspects des soins de soutien doivent faire partie intégrante de la pratique de tous les professionnels de la santé et se concrétiser par des actes tels qu'offrir au patient un soutien émotionnel, fournir de l'information dans le cadre des interactions et aiguiller les patients vers d'autres prestataires, le cas échéant. En plus des soins de soutien prodigués aux patients et à leur famille par les oncologues, les infirmières, les radiothérapeutes et les pharmaciens, un programme complet de soins de soutien intègre les services d'autres intervenants dont les suivants :

Professionnel de la santé : travailleurs sociaux, psychologues, médecins de famille, médecins en soins palliatifs, membres du clergé/aumôniers, diététistes, infirmières spécialisées, physiothérapeutes, ergothérapeutes, orthophonistes et thérapeutes œuvrant dans des domaines tels que les arts, la musique et le toucher;

Tableau 3. Modèle de programme de soins de soutien : il se peut que tous les services ne soient pas offerts dans un même contexte clinique; pour que les patients/familles jouissent d'un accès précoce à la gamme complète des services, il faudra peut-être établir des partenariats entre les programmes de soins en cancer et les organismes communautaires

\begin{tabular}{|c|c|c|c|}
\hline Service ou activité & Groupe cible du service & But du service & $\begin{array}{l}\text { Intervenants } \\
\text { offrant le service }\end{array}$ \\
\hline $\begin{array}{l}\text { Orientation/enseignement } \\
\text { continu au patient/ } \\
\text { à la famille }\end{array}$ & $\begin{array}{l}\text { Tous les nouveaux } \\
\text { patients/proches }\end{array}$ & $\begin{array}{l}\text { Présenter le centre de cancérologie, soins en } \\
\text { cancérologie et le système de cancérologie; offrir de } \\
\text { l'information sur les ressources; fournir des liens } \\
\text { vers des personnes-ressources à des fins de suivi }\end{array}$ & $\begin{array}{l}\text { Personnel/patients } \\
\text { Bénévoles }\end{array}$ \\
\hline $\begin{array}{l}\text { Soutien émotionnel et } \\
\text { information par les pairs } \\
\text { (p. ex. visites dans le } \\
\text { cadre de Toujours femme, } \\
\text { Surmonter le cancer, } \\
\text { groupes d'entraide) }\end{array}$ & $\begin{array}{l}\text { Les personnes qui souhaitent } \\
\text { parler avec un autre patient au } \\
\text { sein d'un groupe ou en face à face }\end{array}$ & $\begin{array}{l}\text { Fournir des occasions de parler, avec d'autres patients } \\
\text { atteints de cancer, de leurs expériences, sentiments, } \\
\text { inquiétudes; partager des expériences et de } \\
\text { l'information entre pairs }\end{array}$ & $\begin{array}{l}\text { Patients atteints } \\
\text { de cancer } \\
\text { Bénévoles }\end{array}$ \\
\hline $\begin{array}{l}\text { Psycho-éducatif } \\
\text { (expérience collective } \\
\text { ou individuelle) }\end{array}$ & $\begin{array}{l}\text { Les personnes qui s'aperçoivent } \\
\text { qu'elles veulent apprendre de } \\
\text { nouvelles habiletés d'adaptation, } \\
\text { exprimer le mal qu'elles ont de } \\
\text { composer avec l'expérience } \\
\text { du cancer }\end{array}$ & $\begin{array}{l}\text { Fournir des occasions d'apprendre des habiletés } \\
\text { d'adaptation et de résolution de problèmes concernant } \\
\text { les enjeux auxquels elles font face }\end{array}$ & $\begin{array}{l}\text { Animateurs de } \\
\text { groupes ayant } \\
\text { reçu une formation } \\
\text { professionnelle }\end{array}$ \\
\hline $\begin{array}{l}\text { Counseling en matière } \\
\text { d'ajustement/de soutien }\end{array}$ & $\begin{array}{l}\text { Les personnes qui ont besoin } \\
\text { d'aide pour s'ajuster à leur } \\
\text { diagnostic et à leur traitement }\end{array}$ & $\begin{array}{l}\text { Fournir aux personnes une aide/un soutien } \\
\text { régulière(er) durant leur traitement }\end{array}$ & Professionnel \\
\hline $\begin{array}{l}\text { Intervention d'urgence } \\
\text { (individuelle) }\end{array}$ & $\begin{array}{l}\text { Les personnes exigeant une } \\
\text { intervention immédiate à propos } \\
\text { de leur détresse émotionnelle, } \\
\text { spirituelle ou psychosociale }\end{array}$ & $\begin{array}{l}\text { Fournir une intervention immédiate visant à } \\
\text { prendre en charge ou à résoudre la détresse } \\
\text { émotionnelle/psychosociale }\end{array}$ & Professionnel \\
\hline $\begin{array}{l}\text { Psychothérapie } \\
\text { - à court terme } \\
\text { - à long terme }\end{array}$ & $\begin{array}{l}\text { Les personnes exigeant une } \\
\text { intervention continue à propos de } \\
\text { leur détresse émotionnelle, } \\
\text { spirituelle ou psychosociale }\end{array}$ & $\begin{array}{l}\text { Fournir une intervention en profondeur continue } \\
\text { visant à prendre en charge ou à résoudre la détresse } \\
\text { émotionnelle/psychosociale }\end{array}$ & Professionnel \\
\hline Intervention nutritionnelle & $\begin{array}{l}\text { Les personnes qui souhaitent être } \\
\text { conseillées en matière de nutrition; } \\
\text { qui éprouvent des difficultés } \\
\text { relativement à l'alimentation }\end{array}$ & $\begin{array}{l}\text { Dispenser des conseils en matière de nutrition / } \\
\text { intervenir auprès des personnes faisant face à des } \\
\text { difficultés d'ordre alimentaire }\end{array}$ & Diététiste \\
\hline $\begin{array}{l}\text { Gestion des symptômes/ } \\
\text { de la douleur }\end{array}$ & $\begin{array}{l}\text { Les personnes qui éprouvent } \\
\text { des difficultés sur le plan de } \\
\text { la gestion de douleur ou } \\
\text { d'autres symptômes (p. ex. } \\
\text { lymphœème, fatigue) }\end{array}$ & Réduire ou éliminer la détresse due aux symptômes & $\begin{array}{l}\text { Professionnel } \\
\text { (p. ex. médecin, } \\
\text { infirmière) }\end{array}$ \\
\hline $\begin{array}{l}\text { Assistance pratique } \\
\text { et fonctionnelle }\end{array}$ & $\begin{array}{l}\text { Les personnes qui ont besoin } \\
\text { d'aide au niveau des activités } \\
\text { quotidiennes, des soins des } \\
\text { enfants; aide financière, aide } \\
\text { pour le transport, etc. }\end{array}$ & $\begin{array}{l}\text { Le service est conçu pour aider à résoudre des } \\
\text { problèmes pratiques (peut se concentrer sur un seul } \\
\text { de ces domaines) et à atteindre un fonctionnement } \\
\text { physique optimal }\end{array}$ & $\begin{array}{l}\text { Personnel } \\
\text { Bénévoles } \\
\text { (selon le } \\
\text { programme) } \\
\text { Professionnel }\end{array}$ \\
\hline
\end{tabular}


Bénévoles : ils offrent du soutien par les pairs, des services de transport et une assistance pratique.

Les patients atteints de cancer reçoivent leurs soins dans de nombreux contextes; cliniques internes, cliniques externes, cabinets de médecin, centres de dépistage et domiciles. Il se peut que les soins de soutien soient nécessaires dans un, plusieurs ou l'ensemble de ces contextes et durant l'entière trajectoire du cancer. Pour que le patient et sa famille reçoivent en temps opportun des soins de soutien appropriés, il faut qu'il existe un réseau d'organismes, de professionnels de la santé et de bénévoles, qui collaborent à la fourniture de l'éventail complet de services. Sans démarche de coordination entre les organismes prodiguant des soins de soutien en cancérologie, les patients et leur famille ne vivront pas l'expérience de soins intégrés ni n'accéderont en temps opportun aux services de soutien. Dans l'ensemble, la coordination des services doit mettre le patient et sa famille au centre d'un réseau de services et d'information.

Des normes fondamentales pour les soins de soutien aux patients atteints de cancer et aux membres de leur famille ont été élaborées et s'appliquent à tous les programmes de cancérologie (Fitch, 1994). Ces normes peuvent être utilisées dans le but de concevoir des approches programmatiques ou d'évaluer le fonctionnement des services (p. ex. fournir des données de référence). Les cinq normes cliniques sont les suivantes :

- Tous les individus reçoivent une évaluation continue en matière de soins de soutien

- Tous les individus ont la possibilité d'être aiguillés vers des ressources appropriées en matière de soins de soutien
- Tous les individus ont le droit d'accéder sans recommandation aux ressources en matière de soins de soutien

- Tous les individus ont accès à une information compréhensible et pertinente sur les aspects médicaux, pratiques et émotionnels de leur cancer et de son traitement;

- Tous les individus reçoivent des soins de soutien qui correspondent à leurs besoins et sont sensibles à leur âge, genre, langue, culture, préférences sexuelles, religion et situation économique.

Le tableau 3 fournit une description des types de services qui peuvent s'avérer bénéfiques pour les patients atteints de cancer et leurs proches, de la raison d'être de chaque service et des intervenants qui participent à leur fourniture. Il n'est pas essentiel que les services incorporés dans un modèle global de soins de soutien soient offerts dans un seul et même contexte clinique. Les services peuvent être offerts par plusieurs organismes qui coopèrent au sein d'une région ou d'un lieu géographiques. Quel que soit le ou les endroit(s) où les services réels sont offerts, le défi fondamental est de s'assurer que les patients et les membres de la famille sont conscients de l'existence des services et qu'ils peuvent y accéder aisément au moment où ils en ont besoin.

La figure 3 présente une manière de conceptualiser la prestation de services en fonction de la proportion des patients qui peuvent avoir besoin de types d'aide particuliers.

\section{Implications sur le plan de l'action}

Au cours de la dernière décennie, des forums de patients (Société canadienne du cancer, 1997; Santé Canada, 1993; National Ovarian

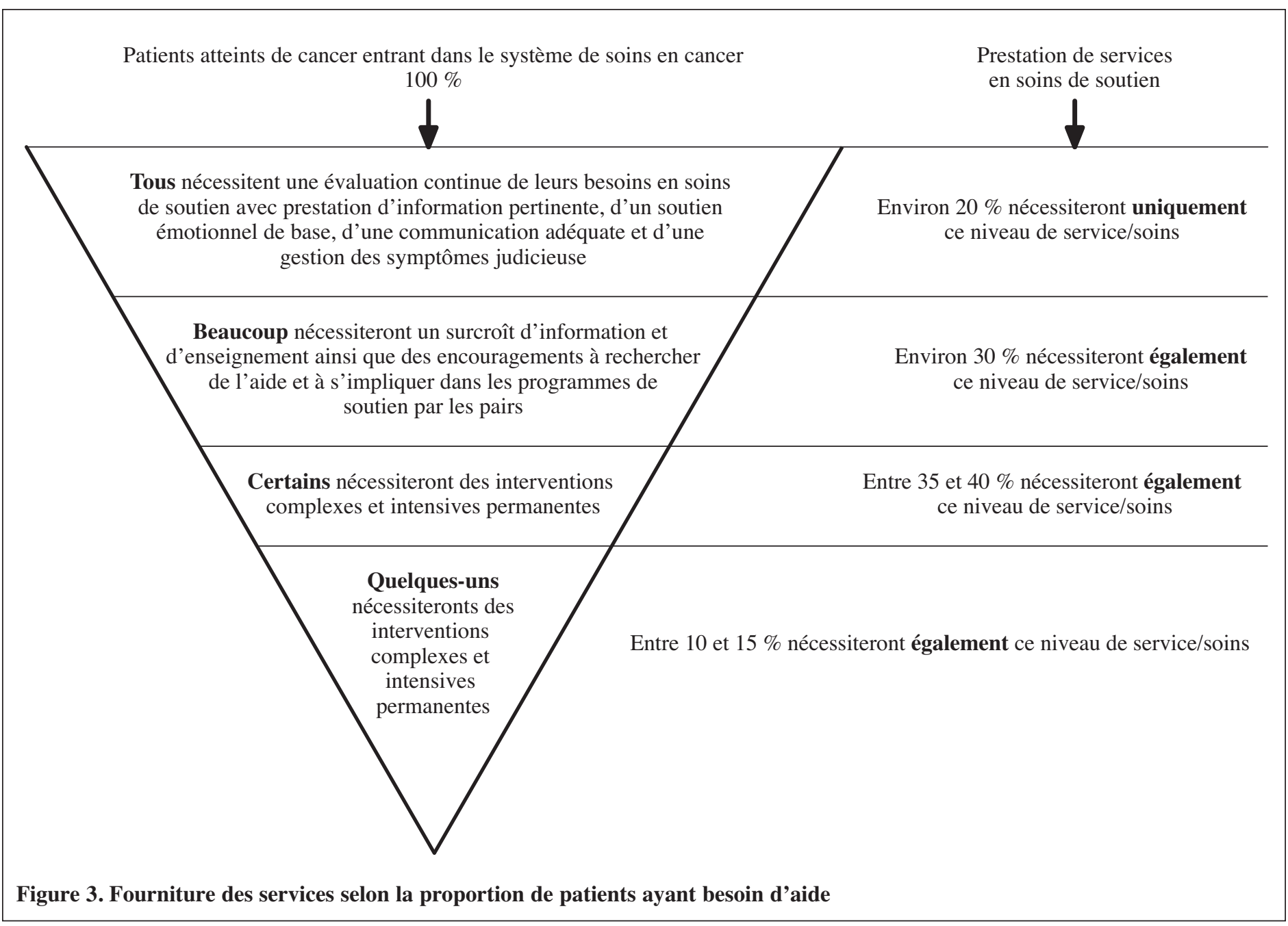


Cancer Association, 1999) et des réunions de parties prenantes (Stratégie canadienne de lutte contre le cancer, 2002; 2005; National Ovarian Cancer Association, 2003-4) ont publié des rapports soulignant les carences de la fourniture des soins de soutien destinés aux patients atteints de cancer et à leurs proches. Une récente enquête nationale auprès de patients atteints de cancer traités en ambulatoire sur leur satisfaction vis-à-vis des soins a révélé que leurs plus grandes inquiétudes concernent la fourniture d'information et de soutien émotionnel (Fitch et Green, 2005). Les patients et les survivants ont lancé des appels passionnés en faveur de l'amélioration du système de soins aux personnes atteintes de cancer. Les types d'améliorations pouvant faciliter l'accès aux soins de soutien comprennent les suivants :

- Incorporer des mécanismes et des processus qui favorisent

l'évaluation de base en soins de soutien, l'information, le soutien, l'aiguillage vers d'autres services, le cas échéant

l'accès aisé au soutien par les pairs et à l'information (groupes d'entraide et de bénévoles)

l'aiguillage aisé et proactif vers des services spécialisés en matière de soins de soutien

- La mise en œuvre de normes de qualité pour les soins de soutien, y compris l'oncologie psychosociale, la réadaptation et les soins palliatifs.

- La mise en œuvre de modèles avec une organisation, une coordination et une intégration améliorées des soins de soutien qui aident les patients et leur famille à naviguer dans le système des soins en cancer

- La création des réseaux de prestataire où l'on a défini des domaines de concentration ou des rôles pour les divers organismes

\section{Références}

Ashbury, F.D., Findley, H., Reynolds, B., \& McKerracher, K. (1998). A Canadian survey of cancer patient experiences: Are their needs being met? Journal of Pain and Symptom Management, 6(5), 298-306.

Bakker, D.A., Fitch, M.I., Gray, R.E., Reed, E., \& Bennett, J. (2001). Patient health care provider communication during chemotherapy: The perspectives of women with breast cancer. Patient Education and Counselling, 43, 61-71.

Bjorck, J.P., Hopp, D.P., \& Jones, L.W. (1999). Prostate cancer and emotional functioning: Effects of mental adjustment, optimism, and appraisal. Journal of Psychosocial Oncology, 17(1), 71-85.

Browne, G.B., Arpin, K., Corey, P., Fitch, M., \& Gafni, A. (1990). Individual correlates of health service utilization and the cost of adjustment to cost adjustment to chronic illness. Medical Care, $18(1), 43-58$.

Bury, M. (1982). Chronic illness as biological disruption. Sociology of Health and Illness, 4(2), July, 167-82.

Canadian Association of Psychosocial Oncology. (2003). The emotional facts of life with cancer. Calgary, AB:Author.

Canadian Cancer Society. (1992). The final report on the needs of persons living with cancer across Canada. Toronto: Author.

Canadian Cancer Society. (1997). Proceedings from the National Forum on Prostate Cancer, Toronto, ON. Toronto: Canada.

Canadian Strategy on Cancer Control. (2002). Proceedings for national stakeholder forum. Toronto, Ontario.

Canadian Strategy on Cancer Control. (2005). Proceedings from national stakeholder forum. Aylmer, Quebec.

Charmaz, K. (1991). Good days, bad days: The self in chronic illness and time. New Brunswick, NJ: Rutgers University Press.

David, H. (1999). Coping with cancer: A personal odyssey. Patient Education and Counseling, 37, 293-297.
- Améliorer l'évaluation des besoins des patients atteints de cancer commencer par les indicateurs de la douleur et de la détresse à titre de processus de dépistage

- Rehausser la formation des professionnels de la santé sur le plan des soins de soutien

- Rehausser la fourniture des services afin de répondre à la demande émanant des patients pour ce qui est de l'oncologie psychosociale, de la réadaptation et des soins palliatifs; examiner qui fournit quels services

- Promouvoir activement le soutien par les pairs et les groupes d'entraide (en collaboration)

- Accroître l'activité de recherche sur des sujets et interventions liés aux soins de soutien

- Examiner les populations de patients particulières sur lesquelles pourraient porter les activités principales (p. ex. patients nécessitant des soins de soutien intensifs-patients atteints au poumon, à la tête et au cou, patients nouvellement diagnostiqués, patients en fin de vie)

\section{Résumé}

Les personnes atteintes de cancer doivent faire face à une myriade de défis. L'adaptation à ces défis peut constituer un fardeau accablant pour les patients et les membres de la famille. Certains individus auront besoin d'aide pour gérer ces défis. Les programmes de soins de soutien permettent d'aider les patients et leur famille à satisfaire l'ensemble des besoins qui peuvent survenir. La fourniture efficace de soins de soutien exige des efforts de coopération de divers organismes et disciplines œuvrant dans un élan commun à l'application d'une approche intégrée de prestation de soins en cancérologie axés sur la personne et sur la compassion.

Davison, B.J., \& Degner, L.F. (1997). Empowerment of men newly diagnosed with prostate cancer. Cancer Nursing, 20(3), 187-96.

Davison, B.J., Degner, L.F., \& Morgan, T.R. (1995). Information and decision-making preferences of men with prostate cancer. Oncology, 22, 1401-8.

Degner, L.F., \& Sloan, J.A. (1995). Symptoms distress in newly diagnosed ambulatory cancer patients as an indicator of survival in lung cancer. Journal of Pain and Symptom Management, 10(6), 423-31.

Dudas, S., \& Carlson, C.E. (1988). Cancer rehabilitation. Oncology Nursing Forum, 15(2), 183-188.

Fitch, M.I. (1994). Providing supportive care for individuals living with cancer (Task Force Report). Toronto: Ontario Cancer Treatment and Research Foundation.

Dudas, S., \& Carlson, C.E. (1988). Cancer rehabilitation. Oncology Nursing Forum, 15(2), 183-188.

Fitch, M.I. (1994). Providing supportive care for individuals living with cancer (Task Force Report). Toronto: Ontario Cancer Treatment and Research Foundation.

Fitch, M.I., Vachon, M., Greenberg, M., \& Franssen, E. (1996). Needs of cancer patients and their family members attending a comprehensive cancer centre. Paper presented in Conference Proceedings, 9th International Cancer Nursing Conference, Brighton, U.K., p. 68.

Fitch, M.I., Gray, R.E., DePetrillo, D., Franssen, E., \& Howell, D. (1999). Canadian women's perspectives on ovarian cancer. Cancer Prevention and Control, 3(1), 52-60.

Fitch, M.I. (2000). Supportive care for cancer patients. Hospital Quarterly, 3(4), 39-46.

Fitch, M., Gowing, J., Murray, A., McKillop, I., Schertzer, L., Deane, K., \& Murray, M. (2002). Understanding supportive care needs 
over time: How well are we doing? 14th MASCC International Symposium, Supportive Care in Cancer, Boston, Massachusetts (June).

Fitch, M., Deane, K. et Howell, D. (2003). Le vécu du cancer ovarien : les perspectives des femmes sur le traitement et la prise de décision thérapeutique. Revue canadienne de soins infirmiers en oncologie, 13(1), 14-20.

Fitch, M.I. (2003). Psychosocial management of patients with recurrent ovarian cancer: Treating the whole patient to improve quality of life. Seminars in Oncology Nursing, 19(3), 40-53.

Fitch, M.I., \& Steele, R. (2003). Supportive care needs of cancer patients: Looking beyond the obvious. Supportive Care Cancer, 11(6), 423.

Fitch, M.I., \& Turner, F. (2004). Canadian stakeholders' perspectives regarding priorities for information dissemination on ovarian cancer. 2 nd National Conference on Ovarian Cancer. Ottawa, Canada (May).

Fitch, M., \& Green, E. (2005). Measuring patient satisfaction across the system. Supportive Care Cancer, 13(6), 472.

Fitch, M.I. (2006). Perspectives of older adults about the cancer information they receive. European Journal of Oncology Nursing, 10(3), 261-7.

Fitch, M.I. (2007). Young women's experiences with breast cancer: An imperative for tailored services. Oncology Nursing Forum, 34(2), 579 (abstract 2470).

Folkman, S., \& Greer, S. (2000). Promoting psychological well-being in the face of serious illness: When theory, research and practice inform each other. Psycho-oncology, 9, 11-19.

Folkman, S., \& Moskowitz, J.T. (2000). Positive affect and the other side of coping. America Psychology, 55(6), 647-54.

Gould, J., James, P., Gray, R.E., \& Fitch, M.I. (2003). Nothing fit me: Cross support needs of young women with breast cancer . PsychoOncology, 12(4), (Suppl), S146-7.

Gray, R.E. (2003). Prostate tales: Men's experiences with prostate cancer. Harriman, TN: Men's Studies Press.

Gray, R.E., Fitch, M.I., Davis, C., \& Phillips, C. (1997). A qualitative study of breast cancer self-help groups. Psychosocial Oncology, 6, 279-89.

Gray, R.E., Greenberg, M., Fitch, M.I., Sawka, C., \& Hampson, A. (1998). Information needs of women with metastatic breast cancer. Cancer Prevention and Control, 2(2), 57-62.

Gray, R.E., Fitch, M.I., Phillips, C., \& Labrecque, M. (2000). Managing the impact of illness: The experiences of men with prostate cancer and their spouses. Journal of Health Psychology, 5, 525-42.

Hack, T.F., Degner, L.F., \& Dyck, D. (1994). Relationship between preferences for decisional control and illness information among women with breast cancer: A quantitative and qualitative analysis. Social Science Medicine, 39, 279-289.

Health Canada (2002). Proceedings from the National Workshop on Palliative Care, Winnipeg, MB, Canada.

Holland, J.C. (1999). NCCN practice guidelines for the management of psychosocial distress. Oncology, 13(5A), 113-147.

Howell, D., Fitch, M.I., \& Deane, K. (2003). Women's experiences with recurrent ovarian cancer. Cancer Nursing, 26(1), 10-17.

Institut national du cancer du Canada (2007). Statistiques canadiennes sur le cancer 2007. Toronto : Auteur.

Kristjanson, L.J., \& Ashcroft, T. (1994). The family's cancer journey: A literature review. Cancer Nursing, 17(1), 1-17.

Lazarus, R., \& Folkman, S. (1984). Stress, appraisal and coping. New York: Springer.

Luker, K.A., Beaver, K., Lenster, S.J., \& Owens, R.G. (1996) Information needs and sources of information for women with breast cancer: A follow-up study. Journal of Advanced Nursing, 23, 487-95.

Maslow, A.H. (1998). Toward a psychology of being (3rd ed.). Mississauga, ON: John Wiley \& Sons Canada Ltd.
McLean, G.L. (1993). Facing death: Conversations with cancer patients. London: Churchill Livingstone.

Muzzin, L.J., Anderson, J.J., Figueredo, A.T., \& Gudelis, S.O. (1994). The experience of cancer. Social Science \& Medicine, 38(9), 1201-8.

National Cancer Institute of Canada. (2005). Strategic plan-2005 report. Toronto: Author.

National Ovarian Cancer Association. (1999). Proceedings from 1st National Forum on Ovarian Cancer, Toronto, Ontario, Canada.

National Ovarian Cancer Association. (2003-4). Stakeholder forums across Canada. Toronto: Ovarian Cancer Information Project.

Park, C.L., \& Folkman, S. (1997). Meaning in the context of stress and coping. Review of General Psychology, 1(2), 115-144.

Parker, J.D.A., \& Endler, N.S. (1996). Coping and defense: A historical overview. In M. Zeidner \& N.S. Endler (Eds.), Handbook of Coping (pp. 3-23).

Pearlin, L.I., \& Schooler, C. (1978). The structure of coping. Journal of Health and Social Behavior, 19(1), 2-21.

Phillips, D. (Ed.). (1998). Heroes: 100 stories of people living with kidney failure. Toronto: Grosvenor House Press.

Pollock, S.E., Christian, B.J., \& Sands, D. (1990). Responses to chronic illness: Analysis of psychological and physiological adaptation. Nursing Research, 39(5), 300-304.

Rosenbaum, E.H. (1982). Living with cancer. New York: Mosby.

Sanson-Fisher, R., Girgis, A., Boyes, A., Bonevski, B., Burton, L., \& Cook, P. (2000). The unmet needs of patients with cancer. Cancer, 88, 217-225.

Santé Canada, 1993. Rapport sur le Forum national sur le cancer du sein, Montréal, Québec, Canada.

Singer, P.A., Margin, D.K., \& Kelner, M. (1999). Quality end-of-life care: Patients' perspectives. Journal of the American Medical Association, 281, 163-168.

Steele, R., Fitch, M.I., Alderson, D., Barrow, K., Hurst, N., \& Doyle, C. (2004). Measuring supportive care needs of women with gynecologic cancer. Supportive Care in Cancer, 12(6), 411.

Société canadienne du cancer (2003). Au-delà des obstacles : Étude des besoins des personnes ayant le cancer et des aidants naturels en Ontario. Toronto : auteur.

Stratégie canadienne de lutte contre le cancer (2002). Disponible à : http://www.partnershipagainstcancer.ca/inside.php?lang=FR\& $\mathrm{pID}=38$

Supportive Care Steering Group. (2004). Supportive care for cancer patients: A forum on collaboration (Greater Toronto Area). Toronto: Author.

Taylor, S.E. (1983). Adjustment to threatening events: A theory of cognitive adaptation. American Psychologist, 38, 1161-73.

Thompson, S.C., \& Collins, M.A. (1995). Applications of perceived control to cancer: An overview of theory and measurement. Journal of Psychosocial Oncology, 13(1/2), 11-26.

Weisman, A.D. (1979). Coping with cancer. New York: McGraw-Hill. Whelan, T.J., Mohide, G.A., Willan, A.R., Arnold, A., Tew, M., Sellick, S. et al. (1997). The supportive care needs of recently diagnosed cancer patients attending a regional cancer centre. Cancer, 81, 1518-1524.

Yates, P. (1999). Family coping: Issues and challenges for cancer nursing. Cancer Nursing, 22(1), 63-71.

Zabora, J.R., Blanchard, C., \& Smith, E.D. (1997). The prevalence of psychological distress across the disease continuum. Journal of Psychosocial Oncology, 15, 75-87.

Zabora, J., BrintzenhofeSzoc, K., Curbow, B., Hooker, C., \& Piantadosi, S. (2001). The prevalence of psychosocial distress by cancer site. Psycho-oncology, 10(1), 19-28.

Zabora, J.R., Loscalzo, M.J., \& Weber, J. (2003). Managing the complications in cancer: Identifying and responding to the patient's perspective. Seminars in Oncology Nursing, 19(4, Suppl. 2), 1-9. 